\title{
Elimination of hepatitis $C$ virus infection in Europe: targeting the obstacles
}

\author{
Dante Romagnoli* \\ Gastroenterology Unit, Polyclinic, Azienda Ospedaliero-Universitaria di Modena, 41124 Modena, Italy
}

*Correspondence: Dante Romagnoli, Gastroenterology Unit, Polyclinic, Azienda Ospedaliero-Universitaria di Modena, 41124 Modena, Italy. dromagno@unimore.it; romagnoli.dante@aou.mo.it

Academic Editor: Lindsay A. Farrer, Boston University School of Medicine, USA

Received: November 23, 2021 Accepted: December 29, 2021 Published: February 24, 2022

Cite this article: Romagnoli D. Elimination of hepatitis C virus infection in Europe: targeting the obstacles. Explor Med. 2022;3:71-6. https://doi.org/10.37349/emed.2022.00075

"It is within the power of man to eradicate infection from the Earth." - Louis Pasteur

\section{Background and aim}

Approximately $1 \%$ of the world's population, namely around 71 million people globally are chronically infected with hepatitis $\mathrm{C}$ virus (HCV), which remains a leading cause of chronic hepatitis, cirrhosis, liver failure and hepatocellular carcinoma on a worldwide basis [1-3].

In 2014, it was estimated that, in Europe, there was a population amounting to 19 million of HCV infected people but this estimate should be corrected based on the limited availability of data from certain areas notably including central and eastern countries [4]. On these grounds, HCV is deemed to be responsible for causing more than 86,000 deaths and 1.2 million disability-adjusted life years (DALYs) in the World Health Organization (WHO) European region and the cause of approximately one in four liver transplants performed in Europe in 2004 [5]. Taken together, these figures are directly and indirectly responsible for a substantial economic burden in Europe comparable to that of the Asia Pacific region and the Americas [6].

In 2016, the WHO set a goal to eliminate HCV as a major public health threat by 2030 through the diagnosis of $90 \%$ of individuals with HCV and initiating treatment in $80 \%$ of those eligible patients [1]. Collectively, these achievements would predictably reduce HCV-related mortality by $65 \%$. Highly effective strategies spanning from prevention to treatment could render these aims accessible. For example, direct-acting antivirals (DAAs), first available in 2014, compared to previously employed interferon (IFN)-based regimens, offer the added values of oral administration, and have reduced duration of treatment, improved tolerability and sustained virologic response rates $>95 \%$ [7]. Nevertheless, so complex and stratified are barriers hampering HCV elimination, that only some European nations (France, Germany, Italy, Spain, Sweden, Switzerland, and the United Kingdom) are on track for meeting the WHO elimination targets by 2030, while others (Austria, Malta and the Netherlands) could only reach these goals by 2040 through expanded screening efforts [8].

With specific reference to recent studies, here I aim at delivering a perspective overview of barriers hampering HCV elimination and how these obstacles can be overcome in Europe. 


\section{Which drawbacks prevent HCV elimination?}

In the pre-SARS-COV-2 era, Mendizabal et al. [9] classified barriers impeding HCV elimination into three different layers: i.e. patient-related; owing to providers; and structural. However, the SARS-CoV-2 epidemic has revolutionized research and practice priorities in the hepatological arena [10]. Accordingly, the stratified complexities that prevent achieving HCV elimination have now become more numerous and difficult to overcome. For example, even before the coronavirus disease 2019 (COVID-19), many countries were already lagging behind to 'catch-up' to elimination targets and, based on a Markov HCV disease burden modeling, a 1-year delay in HCV programs would expectedly result in excess morbidity and mortality owing to HCV infection [11].

With regard to the so-called "macro-elimination" area, the high proportion of undiagnosed and/or unlinked to care patients represents a major challenge. It has recently been estimated that this population at the F0-F3 liver fibrosis stage consists of at least 280,000 individuals [12]. Additionally, "micro-elimination" strategies include targeting populations belonging to a well-defined context (e.g., prison and dialysis unit); specific clinical features [e.g., people living with human immunodeficiency virus (HIV) and people living with blood disorders]; or a given geographic setting (e.g., town/province/nation) [13].

\section{Priority populations}

\section{Immigrants}

Immigrants, that total approximately 50 million residents in Europe, are a challenge that EU countries have to face with respect to achieving HCV elimination [14]. Preliminary data suggest that mirroring rates in the countries of origin, the prevalence of HCV infection among migrants to the EU is higher than that of the native European population [14]. Therefore, although with country-to-country variability, migrants are deemed to be a key group for case finding and treatment [15].

\section{People who inject drugs}

In order to meet the WHO HCV elimination goals by 2030, enhancing prevention, diagnosis, treatment and follow-up among people who inject drugs (PWID) represent an acknowledged priority. The proportion of high-risk drug users receiving opioid substitution therapy in European countries exhibits a substantial country-to-country variability [14]. Similarly, access to community testing, psychiatric or addiction services, social care resources and policy responses are all variable $[16,17]$. Given that PWID are young and, in many cases, have recent infections, they tend to have absent-to-early fibrosis stages $[17,18]$.

\section{Late presenters}

From the perspective of public health providers, delayed presentation for assistance will negatively affect the HCV cascade of care, therefore curbing our attempts to reduce morbidity and mortality resulting from HCV-related liver diseases [19]. Data clearly indicate that individuals with diabetes and/or risky alcohol consumption are prone to the risk of exhibiting "late presentation for HCV care", which is defined as having both an advanced stage of liver fibrosis (or cirrhosis) and being HCV treatment-naive at first presentation for HCV care [20].

Men who have sex with men and other populations targetable for micro-elimination

While the prevalence of sexual transmission of HCV among heterosexuals and HIV-negative men who have sex with men (MSM) is low, the MSM HIV population is at a high risk of HCV infection [21]. The emergence of HCV as an infection transmitted per sexual route among HIV-positive MSM has been well documented in Switzerland, France, the Netherlands, England, Germany, Belgium, and Italy [21, 22]. Therefore, further interventions are needed to reach HCV micro-elimination in MSM living with HIV [23]. Additional categories of individuals who could become the target of micro-elimination strategies include individuals with sexually transmitted diseases (STD), prisoners, HIV and psychiatric patients (Figure 1). 


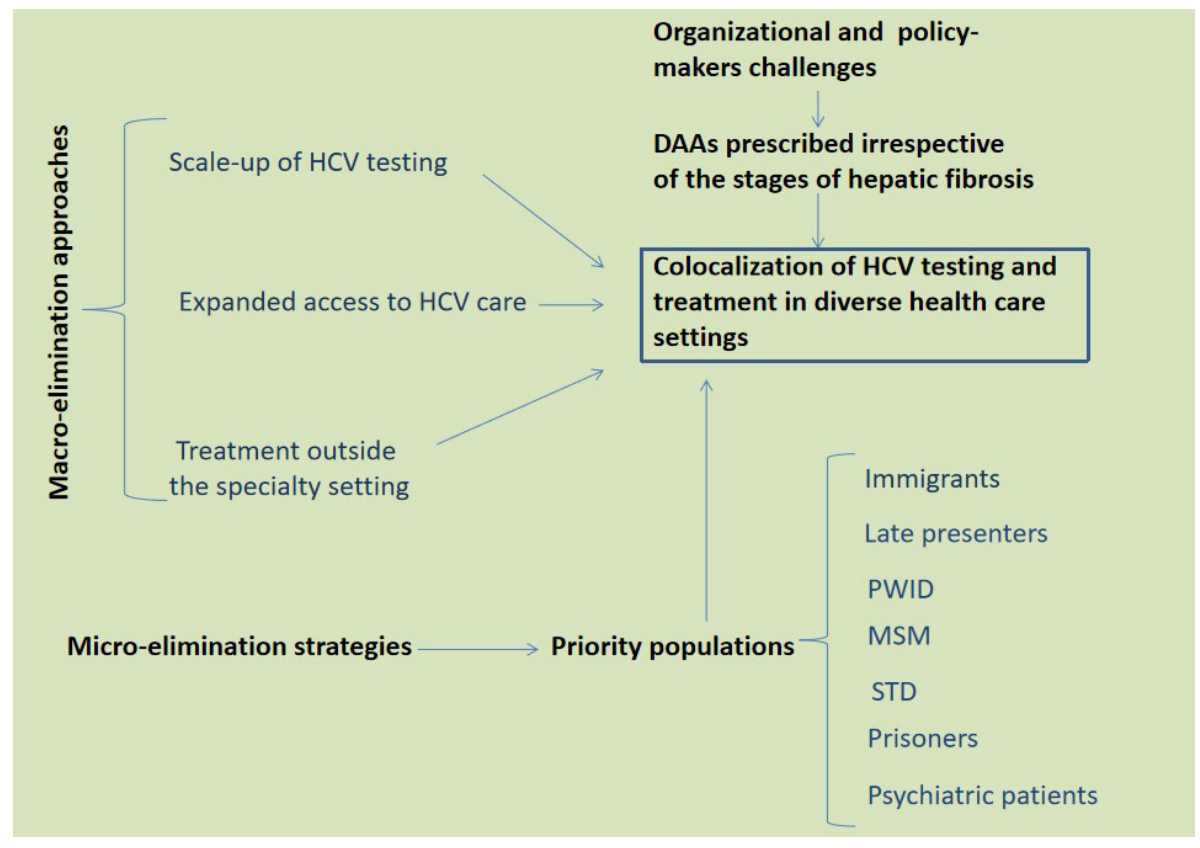

Figure 1. HCV macro- and micro-elimination approaches and strategies. Based on data relevant to the European scenario discussed in the present perspective article as well as the report by the HCV Special Interest Group of the American Association for the Study of the Liver [13], I envisage co-localization of HCV testing and treatment in diverse health care settings as the key to eliminating HCV in Europe. Clearly, this would require a strong input from policy-makers towards the distribution of DAA whenever appropriate, irrespective of liver fibrosis stages, and also outside of the hepatological specialty settings. Further to macro-elimination approaches, special emphasis should be given to well defined priority populations

\section{Differences in pledges to eliminate HCV between autonomous and national health care systems}

In the European Union, country-specific historical, socio-cultural, and economic-political foundations have contributed to determining different health care systems; therefore, considerable variations exist among Member States in terms of the organizational structure of health care, as well as assets of financial investments and allocation of human resources (https://www.europarl.europa.eu/workingpapers/saco/ pdf/101_en.pdf) [24]. Further to this, underfinancing, underdiagnosis, undertreatment, a lack of established national screening programs and limited political commitment to the elimination of HCV has specifically been reported [14]. This occurs in striking variance with joint agreements which have been put in place for pandemic vaccines [14]. These barriers have been identified specifically as obstacles preventing the elimination of HCV as projected by WHO before 2030 in central Europe [25].

\section{COVID-19 pandemics}

Kondili et al. [8] have observed that "All analytic models to assess the progress towards HCV elimination are based on projections to 2030 not including the impact of the COVID-19 pandemic on hepatitis-related services." The COVID-19 pandemic, which has resulted in delayed diagnosis and management of HCV infection, may be transformed from a challenge into an opportunity provided that all those interventions that have been displayed during and after the COVID-19 pandemic are diverted towards increasing diagnosis and linkage to care of people with HCV [26].

\section{How can these barriers be overcome?}

Several approaches may be implemented to target those obstacles that prevent effective elimination of HCV infection.

\section{Diagnosis}

High-risk groups must be targeted in order to implement or reinforce HCV screening and treatment [14]. For example, in this setting, to identify HCV antibody (HCV-Ab), innovative and simpler testing based on either saliva or capillary blood may be used in lieu of the conventional HCV-Ab-based and HCV ribonucleic acid (RNA)-based diagnostic cascade [27]. Therefore, automatic reflex RNA PCR testing following positive 
HCV-Ab test streamlines the screening and testing process [27]. Individuals with undiagnosed active HCV infection need to be identified through screening campaigns conducted among at-risk populations selected based on their birth cohorts [8]. On these grounds, the Italian Ministry of Health has licensed a specific decree (Decreto Milleproroghe 17.12.2020) in order to perform target HCV screening in special populations by December 2022 [28].

\section{Treatment}

Treatment with DAA should be prescribed without any treatment restrictions based on the stages of hepatic fibrosis given that these would detract from the chances of achieving the requested $90 \%$ reduction in incident cases [27]. Additionally, the shorter the duration of treatment the greater the saving of resources so allowing for more individuals to be treated [29].

\section{Organizational and policy-makers challenges}

Migrant populations must be offered full access to health care benefits by removing all differences that separate these individuals from European Union native citizens [14]. Within the European Union boundaries, consistent strategies must be applied in terms of data management, appropriate screening campaigns targeted to the most appropriate populations, pharmacy acquisition and cost containment [14]. To this end, access to affordable generic DAAs must be guaranteed whenever drug pricing is deemed to hamper the elimination of HCV infection [8].

High-prevalence enclaves such as prisons, addiction centers, and certain geographical areas must be offered decentralized care and dedicated point-of-care testing. With reference to the aim of reaching the WHO HCV elimination objective, all those interventions in place during and after the COVID-19 pandemic should be transferred towards enhancing diagnosis and treatment of people with HCV. For example, COVID-19 vaccination hubs could be used to conduct HCV screening such as has been done in Italy with success [26].

\section{Conclusions}

The present perspective article has identified some critical steps that need to be taken in order to achieve the goal to eliminate HCV as a major public health threat by 2030 in Europe. To this end, I have first briefly examined the epidemiological burden of HCV infection. Second, I have re-classified barriers which prevent the WHO-supported aim to eliminate HCV infection by year 2030. As regards patient-related barriers, I have discussed high-hazard cohorts including migrants, PWID and late presenters. These populations deserve special attention for screening and treatment purposes. Next, I have addressed those structural barriers that obstacle the WHO campaign: these include differences between the autonomous health-care systems and limits in national health care system pledges to eliminating HCV in Europe. Finally, in discussing how these barriers may be overcome, emphasis has been put on innovative diagnostic and therapeutic protocols of HCV infection; as well as on how health care providers and policy makers may improve organization aimed at delivering more efficient cures to eliminate HCV infection (Figure 1). Based on the sum of the above notions, I conclude, with Louis Pasteur, "it is within the power of man to eradicate infection from the earth [30]".

\section{Abbreviations}

COVID-19: coronavirus disease 2019

DAAs: direct-acting antivirals

HCV: hepatitis $C$ virus

HCV-Ab: hepatitis C virus antibody

HIV: human immunodeficiency virus

MSM: men who have sex with men

PWID: people who inject drugs

WHO: World Health Organization 


\section{Declarations}

Author contributions

The author contributed solely to the work.

\section{Conflicts of interest}

In the past three years, I attended Advisory Boards for Abbvie and Gilead Sciences.

\section{Ethical approval}

Not applicable.

\section{Consent to participate}

Not applicable.

\section{Consent to publication}

Not applicable.

\section{Availability of data and materials}

Not applicable.

\section{Funding}

Not applicable.

\section{Copyright}

(C) The Author(s) 2022.

\section{References}

1. Global hepatitis report, 2017 [Internet]. Geneva: World Health Organization; c2022 [cited 2021 Dec 19]. Available from: https://www.who.int/publications/i/item/global-hepatitis-report-2017

2. Polaris Observatory HCV Collaborators. Global prevalence and genotype distribution of hepatitis C virus infection in 2015: a modelling study. Lancet Gastroenterol Hepatol. 2017;2:161-76.

3. European Association for the Study of the Liver. EASL recommendations on treatment of hepatitis C 2018. J Hepatol. 2018;69:461-511.

4. Negro F. Epidemiology of hepatitis C in Europe. Dig Liver Dis. 2014;46 Suppl 5:S158-64.

5. Mühlberger N, Schwarzer R, Lettmeier B, Sroczynski G, Zeuzem S, Siebert U. HCV-related burden of disease in Europe: a systematic assessment of incidence, prevalence, morbidity, and mortality. BMC Public Health. 2009;9:34.

6. El Khoury AC, Wallace C, Klimack WK, Razavi H. Economic burden of hepatitis C-associated diseases: Europe, Asia Pacific, and the Americas. J Med Econ. 2012;15:887-96.

7. Pawlotsky JM, Feld JJ, Zeuzem S, Hoofnagle JH. From non-A, non-B hepatitis to hepatitis C virus cure. J Hepatol. 2015;62:S87-99.

8. Kondili LA, Craxì A, Aghemo A. Absolute targets for HCV elimination and national health policy paradigms: foreseeing future requirements. Liver Int. 2021;41:649-55.

9. Mendizabal M, Alonso C, Silva MO. Overcoming barriers to hepatitis C elimination. Frontline Gastroenterol. 2019;10:207-9.

10. Lonardo A, Ballestri S. Perspectives of nonalcoholic fatty liver disease research: a personal point of view. Explor Med. 2020;1:85-107.

11. Blach S, Kondili LA, Aghemo A, Cai Z, Dugan E, Estes C, et al. Impact of COVID-19 on global HCV elimination efforts. J Hepatol. 2021;74:31-6. 
12. Kondili LA, Andreoni M, Alberti A, Lobello S, Babudieri S, Roscini AS, et al. Estimated prevalence of undiagnosed HCV infected individuals in Italy: a mathematical model by route of transmission and fibrosis progression. Epidemics. 2021;34:100442.

13. Feld JJ, Ward JW. Key elements on the pathway to HCV elimination: lessons learned from the AASLD HCV special interest group 2020. Hepatol Commun. 2021;5:911-22.

14. Cooke GS, Andrieux-Meyer I, Applegate TL, Atun R, Burry JR, Cheinquer H, et al; Lancet Gastroenterology \& Hepatology Commissioners. Accelerating the elimination of viral hepatitis: a Lancet Gastroenterology \& Hepatology Commission. Lancet Gastroenterol Hepatol. 2019;4:135-84.

15. Falla AM, Hofstraat SHI, Duffell E, Hahné SJM, Tavoschi L, Veldhuijzen IK. Hepatitis B/C in the countries of the EU/EEA: a systematic review of the prevalence among at-risk groups. BMC Infect Dis. 2018;18:79.

16. Day E, Hellard M, Treloar C, Bruneau J, Martin NK, Øvrehus A, et al; International Network on Hepatitis in Substance Users (INHSU). Hepatitis C elimination among people who inject drugs: challenges and recommendations for action within a health systems framework. Liver Int. 2019;39:20-30.

17. Spina A, Eramova I, Lazarus JV. Policy responses to viral hepatitis B and C among people who inject drugs in Member States of the WHO European region: a sub-analysis of the WHO 2013 global hepatitis policy survey. BMC Infect Dis. 2014;14 Suppl 6:S15.

18. Gamkrelidze I, Pawlotsky JM, Lazarus JV, Feld JJ, Zeuzem S, Bao Y, et al. Progress towards hepatitis C virus elimination in high-income countries: an updated analysis. Liver Int. 2021;41:456-63.

19. Lazarus JV, Picchio C, Dillon JF, Rockstroh JK, Weis N, Buti M. Too many people with viral hepatitis are diagnosed late-with dire consequences. Nat Rev Gastroenterol Hepatol. 2019;16:451-2.

20. Santos M, Protopopescu C, Delarocque-Astagneau E, Bourlière M, Petrov-Sanchez V, Di Beo V, et al; ANRS CO22 HEPATHER study group. Late presentation for HCV care: time to target people with diabetes and/or hazardous alcohol use (ANRS CO22 HEPATHER cohort). Liver Int. 2022;42:38-49.

21. Cuomo G, Digaetano M, Menozzi M, Tagliazucchi S, Guaraldi G, Borghi V, et al. Incidence of HCV infection amongst HIV positive men who had sex with men and prevalence data from patients followed at the Infectious Diseases Clinic of Modena, Italy. Dig Liver Dis. 2018;50:1334-8.

22. Chan DP, Sun HY, Wong HT, Lee SS, Hung CC. Sexually acquired hepatitis C virus infection: a review. Int J Infect Dis. 2016;49:47-58.

23. Castry M, Cousien A, Bellet J, Champenois K, Pialoux G, Yazdanpanah Y, et al; French Hospital Database on HIV (ANRS CO4-FHDH Cohort). Hepatitis $\mathrm{C}$ virus (HCV) incidence among men who have sex with men (MSM) living with HIV: results from the French Hospital Database on HIV (ANRS CO4-FHDH) cohort study, 2014 to 2017. Euro Surveill. 2021;26:2001321.

24. Jakubowski E, Busse R. Health care systems in the EU. A comparative study. Chambers GR, editor. Luxembourg: European Parliament; 1998.

25. Flisiak R, Zarębska-Michaluk D, Frankova S, Grgurevic I, Hunyady B, Jarcuska P, et al. Is elimination of HCV in 2030 realistic in Central Europe. Liver Int. 2021;41 Suppl 1:56-60.

26. Kondili LA, Aghemo A, Andreoni M. Challenges on the achievement of World Health Organization goals for HCV elimination in Italy: need for a regional programmatic approach on screening and linkage to care. Commentary. Ann Ist Super Sanita. 2021;57:201-4.

27. AASLD-IDSA HCV Guidance Panel. Hepatitis C guidance 2018 update: AASLD-IDSA recommendations for testing, managing, and treating hepatitis $C$ virus infection. Clin Infect Dis. 2018;67:1477-92.

28. Mille Proroghe Act, UE EURATOM 2020/2053, Pub n.323 (December 31, 2020).

29. Andreoni M, Di Perri G, Persico M, Marcellusi A, Ethgen O, Sanchez Gonzalez Y, et al. Addressing HCV elimination barriers in Italy: healthcare resource utilization and cost impact using 8 weeks' glecaprevir/ pibrentasvir therapy. Infect Dis Ther. 2021;10:763-74.

30. de Quadros CA. History and prospects for viral disease eradication. Med Microbiol Immunol. 2002;191:75-81. 